\title{
So few women in engineering
}

\section{from Richard Pearson}

As women increasingly become a major force in the labour market, their absence in certain occupations becomes even more of an anachronism.

WOMEN are noticeably absent from many engineering and technological occupations in most countries of the world. The highest representations appear to be in the Soviet Union, where women account for 40 per cent of the engineers, and Republic where they account for 30 per cent. In the United States and the United Kingdom women account for about one in ten of all engineers, rather fewer than in France where the proportion is one in six.

Is the situation changing? The proportion of women graduates has risen in the United States from 3 per cent in 1975 to well over 10 per cent in the $1980 \mathrm{~s}$, and from 4 to 16 per cent in the decade to 1983 in France. In the United Kingdom the proportion of women among engineering graduates has risen from 4 to over 11 per cent during the decade to 1985 .

Although these proportions are increasing as a result of different mixes of chang ing social conditions and policy initiatives in each country, the barriers to the entry of women to the profession seem to be similar across countries. Engineering is not of course a homogeneous profession and the resultant social and attitudinal factors seem to be directing those women that are involved towards chemical and electrical and electronic engineering rather than civil or mechanical engineering - although in the United Kingdom women are poorly represented in electrical/electronic engineering. There are also major differences in enrolments by women in different institutions. One theory to support the latter variability is that a threshold of "minority" enrolments needs to be achieved before steadily increasing enrolments can be established. Thus where enrolments remain at or below 5 per cent, women in non-traditional disciplines are often labelled as "untypical", "unfeminine" and by male peers "different", putting them under pressure and inhibiting growth in enrolments. Women then have to be very single minded to succeed in such a culture. However, where enrolments can be increased to around 15 per cent, for example through the use of targeted or special measures, although women are still seen as a distinct minority, they are more accepted and can generate their own peer group support mechanisms. They then present role modelling examples leading to further growth which, once it reaches a figure of about one third representation, both staff and students regard as being a ba- lanced representation, and minority perceptions and attitudes recede. If this is the case, then it argues for targeted policy initiatives, aimed at concentrating women's representation within a more limited number of institutions so that "critical masses" and "thresholds" can be quickly achieved and then built upon.

Earlier this year the Employment column looked at the role of career breaks in aiding women's employment and careers and commented on their rarity, and the broader social difficulties women face in returning to work (Nature 319, 522; 1986). It showed that there is still considerable resistance to women operating flexible career patterns, and that labour market factors, primarily shortages of skilled staff, are likely to prove a more significant engine of change. However, the latter presumes that women have the basic skills and education to participate in these growth and shortage occupations. Thus while career breaks can only be one relatively minor initiative aimed at helping women participate equally in working life, broader social, educational and employment policies are also needed, some of which have now been operating for a decade or more. In the United States, for example, the 1974 Women Educational Equity Act was enacted as a major initiative aimed at confronting sexism in education. Through the funding of school, college and university programmes, materials and model programmes were designed to help girls achieve educational equity. These funds were split between teachers and administrators, community groups outside the formal education system and the students themselves. Other initiatives have been started, particularly focusing on science and engineering, and a National Science Foundation directory lists over 300 projects designed to increase female participation in non-traditional fields, with the private sector providing almost a third of the funding. The focus of most projects was on engineering, mathematics or general science subjects, and over 80 per cent were based in universities and colleges.

Residential summer schools are also now a common form of initiative with girls attending 2-4 week workshops giving overviews of engineering, specialist teaching in mathematics and computing, industrial field visits and practical experience through project work. Industrial companies are major participants in these programmes providing both general funding and direct assistance in the form of projects, advice and teaching staff. Direct work with schools and students is also seen as important both in generating awareness of careers in engineering and in providing more direct counselling. Within higher education itself, various support schemes operate to help and encourage women to pursue engineering courses, while other schemes seek to encourage them to enrol on engineering courses after more general first-year programmes. More radically, dual degree programmes now exist which enable women in a liberal arts college to transfer to an engineering degree in another college in their third year, by which time they have developed the intellectual and social skills to enable them to participate equally on "male dominated" engineering courses. Finally the establishment of women's professional support groups and the use of female role models are all further helping the process of enhancing women's participation in science and engineering. In the United Kingdom the biggest initiative to date has been Women in Science and Engineering Year in 1984 (Nature 315, 84; 1984) which gave further impetus to a broad range of initiatives already under way which are aimed at raising the profile of engineering and science careers for girls, although there were no significant injections of cash to aid the process. A limited number of scholarships have, however, been paid to help girls acquire the necessary training and further education to become technicians. After a slow start and limited impact, the equal opportunity legislation of the early 1970 s is now at last seen to be having an impact.

In the West at least, women are still significantly under-represented in engineering and technology. Progress is being made under a mixture of market forces and individual initiatives, but it is likely to be some decades before equality is in prospect. The process can only be accelerated by action across a wide range of fronts, including perhaps a national policy commitment, funding for affirmative action programmes, and special counselling and experience schemes aimed at school, higher education and the labour market, as well as in the home and society at large.

Richard Pearson is at the Institute of Manpower Studies, Mantell Building, University of Sussex, Brighton BN1 9RF, UK 